\title{
Stochastic modeling of surface viscoelastic treatments combined with model condensation procedures
}

\author{
A.M.G. de Lima ${ }^{\text {a }}$, D.A. Rade ${ }^{\mathrm{a}, *}$ and N. Bouhaddi ${ }^{\mathrm{b}}$ \\ ${ }^{a}$ Federal University of Uberlândia - School of Mechanical Engineering, Campus Santa Mônica, P.O.Box 593, CEP \\ 38400-902, Uberlândia-MG, Brazil \\ ${ }^{\mathrm{b}}$ University of Franche-Comté, FEMTO-ST UMR 6174, Applied Mechanics Laboratory, 24 Chemin de l'Epitaphe, \\ 25000, Besançon, France
}

\begin{abstract}
Engineering structures incorporating viscoelastic materials are characterized by inherent uncertainties affecting the parameters that control the efficiency of the viscoelastic dampers. In this context, the handling of variability in viscoelastic systems is a natural and necessary extension of the modeling capability of the present techniques of deterministic analysis. Among the various methods devised for uncertainty modeling, the stochastic finite element method has received major attention, as it is well adapted for applications to complex engineering systems. In this paper, the stochastic finite element method applied to a structural three-layer sandwich plate finite element containing a viscoelastic layer, with random parameters modelled as random fields, is presented. Accounting for the dependence of the behaviour of the viscoelastic materials with respect to frequency and temperature, using the concepts of complex modulus and shift factor, the uncertainties are modelled as homogeneous Gaussian stochastic fields and are discretized according to the spectral method, using Karhunen-Lœève expansions. The modeling procedure is confined to the frequency domain, and the dynamic responses are characterized by frequency response functions (FRF's). Monte Carlo Simulation (MCS) combined with Latin Hypercube Sampling is used as the stochastic solver. The typically high dimensions of finite element models of viscoelastic systems combined with the large number of Monte Carlo samples to be computed make the evaluation of the FRF's variability computer intensive. Those difficulties motivate the use of condensation methods specially adapted for viscoelastic systems, in order to alleviate the computational cost. After the presentation of the underlying formulation, numerical applications of moderate complexity are presented and discussed aiming at demonstrating the main features and, particularly, the computation cost savings provided by the association of MCS with the suggested condensation procedure.
\end{abstract}

Keywords: Uncertainty propagation, viscoelasticity, model condensation, stochastic finite elements

\section{Introduction}

In the context of passive control of mechanical vibrations, much effort has been devoted to the development of finite element models capable of accounting for the typical dependence of the viscoelastic behavior with respect to frequency and temperature [1-5]. A natural extension of the deterministic modeling capability is to account for the uncertainties in the design variables aiming at evaluating the influence of the variability on the model predictions [69]. Such uncertainty propagation analysis becomes especially interesting for application to viscoelastic systems, since the mechanical properties of such materials are strongly dependent on a certain number of operational and environmental factors, among which frequency and temperature are considered to be the most important [10].

\footnotetext{
*Corresponding author: E-mail: domingos @ufu.br.
} 
The stochastic finite element method (SFEM), in association with Monte Carlo Simulation (MCS) have been applied to assess response variability in a broad range of linear and non-linear static and dynamic problems [11-13]. Most of those studies make clear that one of the main limitations of such strategy is the high computational cost which results from the use of FE models containing large numbers of DOFs and the necessity of computing a large number of response samples to achieve the necessary statistical significance. Moreover, few works, such the one in reference [14], have addressed the influence of uncertainties on the response variability of viscoelastic systems, in which ad-hoc procedures must be used to cope with the frequency- and temperature-dependence of the behavior of viscoelastic materials.

The present paper describes a complete methodology for uncertainty propagation which combines: (a) a threelayer sandwich plate stochastic viscoelastic finite element, previously described in reference [14], for which a parameterization approach has been suggested in such a way to enable the introduction of parametric variations in a straightforward manner; (b) a model condensation strategy specially adapted to viscoelastic systems, in which the dynamic response of the damped system is projected on a truncated modal basis of the associated undamped system, such basis being enriched by static residual vectors; (c) MCS combined with Latin Hypercube (LHC) sampling [15] as the stochastic solver used to evaluate the response variability.

\section{Introduction of the viscoelastic behavior into finite element models}

In this paper, as the interest is confined to frequency-domain analyses, the so-named Complex Modulus approach is used in combination with the Frequency-Temperature Correspondence Principle and the Elastic-Viscoelastic Correspondence Principle [10].

According to the Complex Modulus approach, the dynamic behavior of viscoelastic materials in the frequency domain is accounted for by using a frequency- and temperature-dependent complex material moduli as follows:

$$
G(\omega, T)=G^{\prime}(\omega, T)\left[l+i \eta_{G}(\omega, T)\right]
$$

where $\omega$ and $T$ denote, respectively, the excitation frequency and the temperature of the viscoelastic material, $G^{\prime}(\omega, T)$ and $\eta_{G}(\omega, T)$ designate, respectively, the so-named storage modulus and loss factor. It should be noted that definition Eq. (1) applies to both longitudinal and transverse moduli $E(\omega, T)$ and $G(\omega, T)$. Moreover, it has been assumed by some authors [1-3,16-18], that the Poisson ratio is independent from frequency and temperature in such a way that the longitudinal and transverse moduli are related to each other through the relation $G(\omega, T)=E(\omega, T) /[2(1+\omega)]$. Such assumption, which has been adopted in this study, has been argued in reference [19], in which the author has verified experimentally the variations of the Poisson ratio for PVC specimens.

The Frequency-Temperature Superposition Principle [10] establishes the equivalence between the effects of the excitation frequency and of the temperature on the properties of a broad class of viscoelastic materials. This implies that the viscoelastic characteristics at different temperatures can be related to each other by changes (or shifts) in the actual values of the excitation frequency. This leads to the concepts of shift factor and reduced frequency, symbolically expressed as:

$$
G(\omega, T)=G\left(\omega_{r}, T_{0}\right)=G\left(\alpha_{T} \omega, T_{0}\right), \quad \eta_{G}(\omega, T)=\eta_{G}\left(\omega_{r}, T_{0}\right)=\eta_{G}\left(\alpha_{T} \omega, T_{0}\right)
$$

where $T$ indicates an arbitrary value of the temperature, $T_{0}$ is a reference value of temperature, $\omega_{r}=\alpha_{T}(T) \omega$ is the reduced frequency, $\omega$ is the actual excitation frequency, and $\alpha_{T}(T)$ is the shift function. Functions $G\left(\omega_{r}\right)$ and $\alpha_{T}(T)$ can be obtained from experimental tests for specific viscoelastic materials [10]. Drake and Soovere [20] suggest analytical expressions for the complex modulus and shift factor for various commercial viscoelastic materials. Equations (3) represent the complex modulus and shift factor defined in the intervals $210 \leqslant T \leqslant 360 \mathrm{~K}$ and $1.0 \leqslant$ $\omega \leqslant 1.0 \times 1.0^{6} \mathrm{~Hz}$, for the $3 \mathrm{M}^{\mathrm{TM}}$ ISD1 12 viscoelastic material, as provided by those authors. The $3 \mathrm{M}^{\mathrm{TM}}$ ISD112 is a rubber-like viscoelastic polymer which is provided by the manufacturer in the form of adhesive tapes [21].

$$
G\left(\omega_{r}\right)=B_{1}+B_{2} /\left(1+B_{5}\left(i \omega_{r} / B_{3}\right)^{-B_{6}}+\left(i \omega_{r} / B_{3}\right)^{-B_{4}}\right)
$$




$$
\log \left(\alpha_{T}\right)=a\left(\frac{1}{T}-\frac{1}{T_{0}}\right)+2.303\left(\frac{2 a}{T_{0}}-b\right) \log \left(\frac{T}{T_{0}}\right)+\left(\frac{b}{T_{0}}-\frac{a}{T_{0}^{2}}-S_{A Z}\right)\left(T-T_{0}\right)
$$

where:

$$
\begin{aligned}
& B_{1}=0.4307 M p a ; B_{2}=1200 M p a ; B_{3}=1543000 ; B_{4}=0.6847 ; B_{5}=3.241 ; B_{6}=0.18 \\
& T_{0}=290 K ; T_{L}=210 K ; T_{H}=360 K ; S_{A Z}=0.05956 K^{-1} ; S_{A L}=0.1474 K^{-1} ; S_{A H}=0.009725 K^{-1} \\
& C_{A}=\left(1 / T_{L}-1 / T_{0}\right)^{2} ; C_{B}=\left(1 / T_{L}-1 / T_{0}\right) ; C_{C}=\left(S_{A L}-S_{A Z}\right) ; D_{A}=\left(1 / T_{H}-1 / T_{0}\right)^{2} ; D_{B}=\left(1 / T_{H}-1 / T_{0}\right) \\
& D_{C}=\left(S_{A H}-S_{A Z}\right) ; D_{E}=\left(D_{B} C_{A}-D_{A} C_{B}\right) ; a=\left(D_{B} C_{C}-C_{B} D_{C}\right) / D_{E} ; b=\left(D_{C} C_{A}-C_{C} D_{A}\right) / D_{E}
\end{aligned}
$$

According to the Elastic-Viscoelastic Correspondence Principle [10] the derivation of the finite element model accounting for the viscoelastic behavior can be carried-out in two distinct phases: first, the element and global stiffness matrices are obtained by considering pure elastic behavior (i.e., frequency- and temperature-independent material moduli), accounting for the strain state assumed by the underlying theory; then, the material moduli are modified to account for the viscoelastic behavior according to the model expressed by Eq. (1). Clearly, this approach leads to frequency- and temperature-dependent finite element stiffnesses matrices. Assuming isotropic behavior and frequency- and temperature-independent Poisson ratio, the global equations of motion in the frequency domain of a viscoelastic structure containing $N$ DOFs, for which one of the moduli has been factored-out of the viscoelastic matrices, can be expressed as follows [18]:

$$
\left[\boldsymbol{K}_{e}+G(\omega, T) \overline{\boldsymbol{K}}_{v}-\omega^{2} \boldsymbol{M}\right] \boldsymbol{Q}(\omega, T)=\boldsymbol{F}(\omega)
$$

where $M \in R^{N \times N}$ is the mass matrix, $K_{e} \in R^{N \times N}$ is the stiffness matrix corresponding to the purely elastic parts, and $\overline{\boldsymbol{K}}_{v} \in R^{N \times N}$ is the frequency- and temperature-independent part of the viscoelastic stiffness matrix. $\boldsymbol{Q}(\omega, T) \in R^{N}$ and $\boldsymbol{F}(\omega) \in R^{N}$ are, respectively, the vectors of the amplitudes of the harmonic generalized displacements and external loads.

The receptance or frequency response function matrix is expressed as:

$$
\boldsymbol{H}(\omega, T)=\left[\boldsymbol{K}_{e}+G(\omega, T) \overline{\boldsymbol{K}}_{v}-\omega^{2} \boldsymbol{M}\right]^{-1}
$$

The computation of the receptance matrix by direct inversion of the dynamic stiffness matrix, as indicated in Eq. (5), is unfeasible in practical situations in which FE models with large numbers of DOFs are dealt with. Thus, alternative procedures for such computation, based on model reduction, will be considered later on to alleviate the computational cost.

\section{Deterministic finite element formulation of a three-layer sandwich plate}

In this section, the model of a moderately thin three-layer sandwich plate finite element, which can be frequently found, for example, in aerospace systems, is summarized, based on the original developments made by Khatua and Cheung [22] and implementation by Lima et al. [16]. Figure 1 depicts a rectangular element formed by an elastic base-plate (1), a viscoelastic core (2) and an elastic constraining layer (3). This element contains four nodes and seven DOF's per node, representing the in-plane displacements in the middle plane of the base-plate in directions $x$ and $y$ (denoted by $u_{1}$ and $v_{1}$, respectively), the in-plane displacements of the middle plane of the constraining layer in directions $x$ and $y$ (denoted by $u_{3}$ and $v_{3}$, respectively), the transverse displacements, $w$, and the cross-section rotations about $x$ and $y$, denoted by $\theta_{x}$ and $\theta_{y}$, respectively.

In the development of the theory, the following assumptions are adopted: (i) all the materials involved are homogeneous and isotropic and present linear mechanical behavior; (ii) normal stresses and strains in direction $z$ are neglected for all the three layers; (iii) the elastic layers (1) and (3) are modeled according to Kirchhoff's theory; (iv) for the viscoelastic core, Mindlin's theory is adopted (transverse shear is included); $(v)$ the transverse displacement $w$ and cross-section rotations $\theta_{x}$ and $\theta_{y}$, are the same for all the three layers.

The strain-displacement relations are used and the resulting strains for each layer is separated by uncoupling membrane, bending and shear effects, respectively, as follows:

For the elastic layers $(k=1,3)$ : 


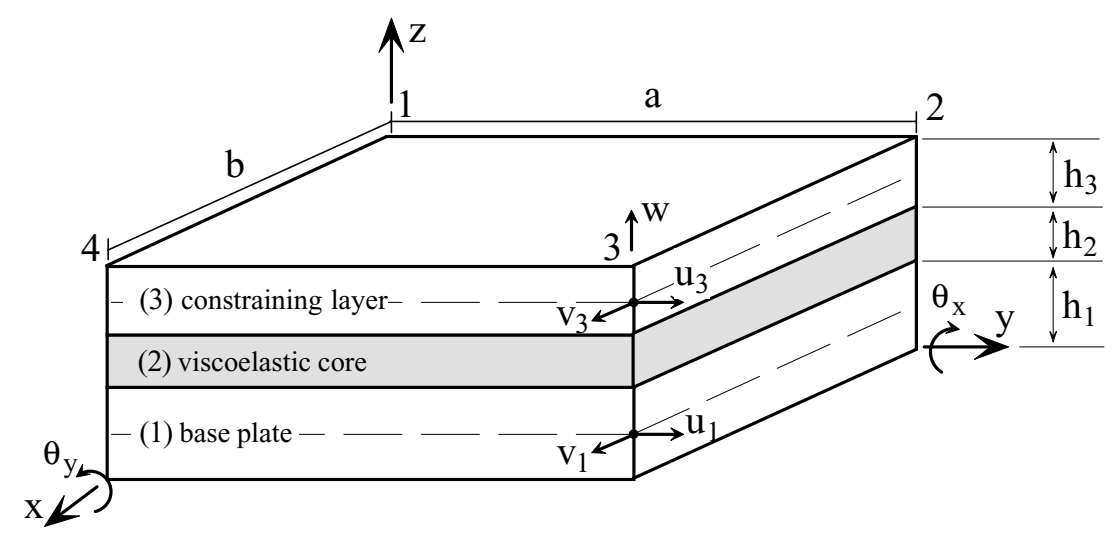

Fig. 1. Illustration of the three-layer sandwich plate element.

$$
\varepsilon^{(k)}=\left[\varepsilon_{x}^{(k)} \varepsilon_{y}^{(k)} \gamma_{x y}^{(k)}\right]^{T}=\varepsilon_{m}^{(k)}+z \varepsilon_{b}^{(k)}
$$

For the viscoelastic core $(v=2)$ :

$$
\varepsilon^{(v)}=\left[\varepsilon_{x}^{(v)} \varepsilon_{y}^{(v)} \gamma_{x y}^{(v)} \gamma_{x z}^{(v)} \gamma_{y z}^{(v)}\right]^{T}=\varepsilon_{m}^{(v)}+\frac{z}{h_{2}} \varepsilon_{b}^{(v)}+\frac{1}{h_{2}} \varepsilon_{s}^{(v)}
$$

In the equations above, subscripts $m, b$ and $s$ designate membrane, bending and shear effects, respectively.

The discretization of the displacement fields within the element is made by using the following linear and cubic interpolation functions:

$$
\begin{aligned}
& u_{1}=a_{1}+a_{2} x+a_{3} y+a_{4} x y \quad u_{3}=a_{9}+a_{10} x+a_{11} y+a_{12} x y \\
& v_{1}=a_{5}+a_{6} x+a_{7} y+a_{8} x y \quad v_{3}=a_{13}+a_{14} x+a_{15} y+a_{16} x y \\
& w=b_{1}+b_{2} x+b_{3} y+b_{4} x^{2}+b_{5} x y+b_{6} y^{2}+b_{7} x^{3}+b_{8} x^{2} y+b_{9} x y^{2}+b_{10} y^{3}+b_{11} x^{3} y+b_{12} x y^{3}
\end{aligned}
$$

Based on the kinematic hypotheses and the stress-states assumed for each layer, the stress-strain relations are obtained, and the strain and kinetic energies of the three-layer sandwich plate finite element are formulated as follows:

$$
\boldsymbol{U}_{(e)}=\frac{1}{2} \boldsymbol{\delta}(t)^{T}\left[\boldsymbol{K}_{e}^{(e)}+\boldsymbol{K}_{v}^{(e)}\right] \boldsymbol{\delta}(t) ; \quad \boldsymbol{T}^{(e)}=\frac{1}{2} \dot{\boldsymbol{\delta}}(t)^{T} \boldsymbol{M}^{(e)} \dot{\boldsymbol{\delta}}(t)
$$

where:

$$
\boldsymbol{M}^{(e)}=\sum_{k=1}^{3} \rho_{k} h_{k} \int_{x=0}^{a} \int_{y=0}^{b} \boldsymbol{N}^{T}(x, y) \boldsymbol{N}(x, y) d y d x
$$

is the elementary mass matrix, and $\boldsymbol{K}_{e}^{(e)}=\boldsymbol{K}_{1}^{(e)}+\boldsymbol{K}_{3}^{(e)}$ and $\boldsymbol{K}_{v}^{(e)}=\boldsymbol{K}_{2}^{(e)}(\omega, T)$ are, respectively, the contributions of the purely elastic and viscoelastic parts of the structure to the elementary stiffness matrix, defined, respectively, as follows:

$$
\begin{aligned}
& \boldsymbol{K}_{e}^{(e)}=\sum_{k=1,3} \int_{z=0}^{h_{k}} \int_{x=0}^{a} \int_{y=0}^{b} \boldsymbol{D}_{k}^{T}(x, y, z) \boldsymbol{C}_{k} \boldsymbol{D}_{k}(x, y, z) d y d x d z \\
& \boldsymbol{K}_{v}^{(e)}(\omega, T)=\int_{z=0}^{z=h_{2}} \int_{x=0}^{x=a} \int_{y=0}^{y=b} \boldsymbol{D}_{v}^{T}(x, y, z) \boldsymbol{C}_{v}(\omega, T) \boldsymbol{D}_{v}(x, y, z) d y d x d z
\end{aligned}
$$


where $h_{k}$ and $\rho_{k}$ are the thickness and the mass density of the $k$-th layer, respectively. Matrices $\boldsymbol{D}_{k}(x, y)(k=1,2,3)$ are formed by differential operators appearing in the strain-displacement relations, as detailed in reference [16]. $C_{k}$ and $\boldsymbol{C}_{v}(\omega, T)$ are, respectively, the matrices of isotropic elastic and viscoelastic material properties.

From the elementary matrices computed for each element of the finite element mesh, the global equations of motion are constructed accounting for node connectivity, using standard finite element assembling procedures.

\section{Parameterization of the finite element model}

At this point it is important to consider that, in order to study the system behavior when uncertainties are present, the random responses have to be computed with respect to a set of uncertain geometrical and physical parameters associated to the three-layer sandwich plate. In general, such random variables intervene in a rather complicated manner in the FE matrices. Hence, in order to evaluate the variability of the responses associated to these uncertainties, it becomes interesting to perform a parameterization of the FE model, which is understood as a means of making the design parameters factored-out of the matrices. At the expense of lengthy algebraic manipulations, this procedure enables to introduce not only the uncertainties into the model, but also to perform a sensitivity analysis in a straightforward way, leading to significant cost savings in iterative optimization or model updating processes.

According to the theory of the sandwich plate FE summarized in Section 3, the design parameters of mass and stiffness of each layer can be factored-out of the elementary matrices by uncoupling membrane, bending and shear effects, respectively, as follows [23]:

$$
\begin{aligned}
\boldsymbol{M}_{1}^{(e)}= & \rho_{1} h_{1 m} \boldsymbol{M}_{1.1}^{(e)}+\rho_{1} h_{1 b} \boldsymbol{M}_{1.1}^{(e)} \\
\boldsymbol{M}_{2}^{(e)}= & \rho_{2} h_{2}{ }_{m} \boldsymbol{M}_{2.0}^{(e)}+\rho_{2} h_{2} d_{1 m} \boldsymbol{M}_{2.1}^{(e)}+\rho_{2} h_{2} d_{1 m}^{2} \boldsymbol{M}_{2.2}^{(e)}+\rho_{2} h_{2 b} \boldsymbol{M}_{2.0}^{(e)} \\
\boldsymbol{M}_{3}^{(e)}= & \rho_{3} h_{3}{ }_{m} \boldsymbol{M}_{3.1}^{(e)}+\rho_{3} h_{3 b} \boldsymbol{M}_{3.1}^{(e)} \\
\boldsymbol{K}_{1}^{(e)}= & E_{1} h_{1 m} K_{1.1}^{(e)}+E_{1} h_{1 b}^{3} \boldsymbol{K}_{1.1}^{(e)} \\
\overline{\boldsymbol{K}}_{2}^{(e)}= & h_{2}\left({ }_{m} \overline{\boldsymbol{K}}_{2.0}^{(e)}+d_{1 m} \overline{\boldsymbol{K}}_{2.1}^{(e)}+d_{1 m}^{2} \overline{\boldsymbol{K}}_{2.2}^{(e)}\right)+h_{2}\left({ }_{b} \overline{\boldsymbol{K}}_{2.0}^{(e)}+d_{2 b} \overline{\boldsymbol{K}}_{2.1}^{(e)}+d_{2}^{2}{ }_{b} \overline{\boldsymbol{K}}_{2.2}^{(e)}\right)+\ldots \\
& \frac{1}{h_{2}}\left({ }_{s} \overline{\boldsymbol{K}}_{2.0}^{(e)}+d_{3{ }_{s}} \overline{\boldsymbol{K}}_{2.1}^{(e)}+d_{3}^{2} d_{2 s}^{2} \overline{\boldsymbol{K}}_{2.2}^{(e)}\right) \\
\boldsymbol{K}_{3}^{(e)}= & E_{3} h_{3}{ }_{m} \boldsymbol{K}_{3.1}^{(e)}+E_{3} h_{3 b}^{3} \boldsymbol{K}_{3.1}^{(e)}
\end{aligned}
$$

where $d_{1}=h_{3}-h_{1}, d_{2}=h_{1}-h_{3}$ and $d_{3}=h_{1}+2 h_{2}+h_{3} \cdot E_{k}(k=1,3)$ designate the longitudinal moduli of the elastic layer. Subscripts $m, b$ and $s$, respectively, indicate the membrane, bending and shear effects in the structural matrices.

\section{Stochastic finite element formulation}

In order to model the system behavior when uncertainties are present, the design parameters which have been factored-out of the matrices appearing in Eq. (10) are taken as random. In this paper, the well-known KarhunenLoève (KL) decomposition, which is a continuous representation for random fields expressed as the superposition of orthogonal random variables weighted by deterministic spatial functions, is used. According to this technique, a random field can be viewed as a spatial extension of a random variable that describes the spatial correlation of a structural parameter that fluctuates randomly. A one-dimensional random field $H(x, \theta)$ is defined by its mean, $E(x)=\varepsilon[H(x, \theta)]$, and its covariance $C\left(x_{1}, x_{2}\right)=\varepsilon\left\{\left[H\left(x_{1}, \theta\right)-E\left(x_{1}\right)\right]\left[H\left(x_{2}, \theta\right)-E\left(x_{2}\right)\right]\right\}$ where $x$ denotes the spatial dependence of the field, $\theta$ represents a random process, and $\varepsilon(\bullet)$ indicates the expectation operator. For a two-dimensional homogeneous Gaussian random field $H(x, y, \theta)$ with a symmetric and positive- 
definite covariance function over a domain $\Omega \in R^{d}$, it is possible to find a unique projection of $H(x, y, \theta)$ on an orthonormal truncated random basis as follows [11]:

$$
H(\boldsymbol{x}, \boldsymbol{y}, \theta)=E(\boldsymbol{x}, \boldsymbol{y})+\sum_{r=1}^{n} \sqrt{\lambda_{r}} f_{r}(x, y) \xi_{r}(\theta)
$$

where the deterministic functions $f_{r}(x, y)$ and the scalar values $\lambda_{r}$ are, respectively, the eigenfunctions and the eigenvalues of the covariance function $C\left[\left(x_{1}, y_{1}\right),\left(x_{2}, y_{2}\right)\right]$.

The KL expansion is defined with reference to a particular geometric domain $\Omega$, so that in the case of modeling an uncertain parameter of a structural model by means of a random field, this geometry at least includes the domain of the structure under consideration.

For some relatively simple geometric configurations, such as the rectangular three-layer sandwich plate element shown in Fig. 1, the analytical solution to the eigenproblem proposed by Ghanem and Spanos [11] for the KL expansion into the domains $\Omega_{x}=\left(x_{1}, x_{2}\right)=[-a, a]$ and $\Omega_{y}=\left(y_{1}, y_{2}\right)=[-b, b]$, is given as follows:

$$
C\left[\left(x_{1}, y_{1}\right),\left(x_{2}, y_{2}\right)\right]=\exp \left(-\frac{\left|x_{1}-x_{2}\right|}{L_{c o r, x}}-\frac{\left|y_{1}-y_{2}\right|}{L_{c o r}, y}\right)
$$

where $L_{c o r, x}$ and $L_{c o r, y}$ represent the correlation lengths in $x$ and $y$ directions, respectively. Taking into account the separability property of the covariance function, the two-dimensional problem is decoupled into two one-dimensional independent eigenproblems with solution $f_{r}(x, y)=f_{i}(x) f_{j}(y)$ and $\lambda_{r}=\lambda_{i} \lambda_{j}$. The couples $\left(\lambda_{i}, f_{i}(x)\right)$ are obtained by solving the KL decomposition of a first-order field of variance equal to one, with a correlation length $L_{c o r, x}$ on a domain $[-a, a]$, while the couples $\left(\lambda_{j}, f_{j}(y)\right)$ are obtained by solving the same problem, but with a correlation length $L_{c o r, y}$ on a domain $[-b, b]$. The procedure for computing such couples is summarized in the following, and details can be found in reference [11]:

$$
\text { For } i \text { and } j \text { odd, with } i \geqslant 1 \text { and } j \geqslant 1 \text { : }
$$

$$
\begin{aligned}
& \lambda_{i}=\frac{2 c_{1}}{\omega_{i}^{2}+c_{1}^{2}}, \quad f_{i}(x)=\alpha_{i} \cos \left(\omega_{i} x\right) \\
& \lambda_{j}=\frac{2 c_{2}}{\omega_{j}^{2}+c_{2}^{2}}, \quad f_{j}(y)=\alpha_{j} \cos \left(\omega_{j} y\right)
\end{aligned}
$$

where $\alpha_{i}=1 / \sqrt{a+\sin \left(2 \omega_{i} a\right) / 2 \omega_{i}}, \alpha_{j}=1 / \sqrt{b+\sin \left(2 \omega_{j} b\right) / 2 \omega_{j}}, c_{1}=1 / L_{c o r, x}, c_{2}=1 / L_{c o r, y}$.

The roots $\omega_{i}$ and $\omega_{j}$ are the solutions of the following transcendental functions:

$$
c_{1}-\omega_{i} \tan \left(\omega_{i} a\right)=0, \quad c_{2}-\omega_{j} \tan \left(\omega_{j} b\right)=0
$$

defined into the domains $\left[(i-1) \frac{\pi}{a},\left(i-\frac{1}{2}\right) \frac{\pi}{a}\right]$ and $\left[(j-1) \frac{\pi}{b},\left(j-\frac{1}{2}\right) \frac{\pi}{b}\right]$, respectively.

For $i$ and $j$ even, with $i \geqslant 2$ and $j \geqslant 2$ :

$$
\begin{aligned}
& \lambda_{i}=\frac{2 c_{1}}{\omega_{i}^{2}+c_{1}^{2}}, \quad f_{i}(x)=\alpha_{i} \sin \left(\omega_{i} x\right) \\
& \lambda_{j}=\frac{2 c_{2}}{\omega_{j}^{2}+c_{2}^{2}}, \quad f_{j}(y)=\alpha_{j} \sin \left(\omega_{j} y\right)
\end{aligned}
$$

where $\alpha_{i}=1 / \sqrt{a-\sin \left(2 \omega_{i} a\right) / 2 \omega_{i}}$ and $\alpha_{j}=1 / \sqrt{b-\sin \left(2 \omega_{j} b\right) / 2 \omega_{j}}$.

The roots $\omega_{i}$ and $\omega_{j}$ are the solutions of the following transcendental functions:

$$
\omega_{i}+c_{1} \tan \left(\omega_{i} a\right)=0 ; \quad \omega_{j}+c_{2} \tan \left(\omega_{j} b\right)=0
$$


defined into the domains $\left[\left(i-\frac{1}{2}\right) \frac{\pi}{a}, i \frac{\pi}{a}\right]$ and $\left[\left(j-\frac{1}{2}\right) \frac{\pi}{b}, j \frac{\pi}{b}\right]$, respectively.

For illustration, Fig. 2 represents the graphs of the first four eigenfunctions of the covariance Eq. (12) for two cases: first, by using $L_{c o r, x}=0.05 \mathrm{~m}$ and $\Omega_{x}=[-0.05,0.05]$; and second, by considering $L_{c o r, x}=0.01 \mathrm{~m}$ and $\Omega_{x}=[-0.01,0.01]$. The first four eigenvalues of the covariance function are: for the first case: $\lambda_{1}=0.03694$, $\lambda_{2}=0.0069, \lambda_{3}=0.00225, \lambda_{4}=0.0011$; for the second case: $\lambda_{1}=0.01872, \lambda_{2}=0.0018, \lambda_{3}=0.00025, \lambda_{4}=$ 0.00022 .

It can be noted that both the correlation length of the random field and the length of the definition domain do influence the eigenvalues and eigenfunctions.

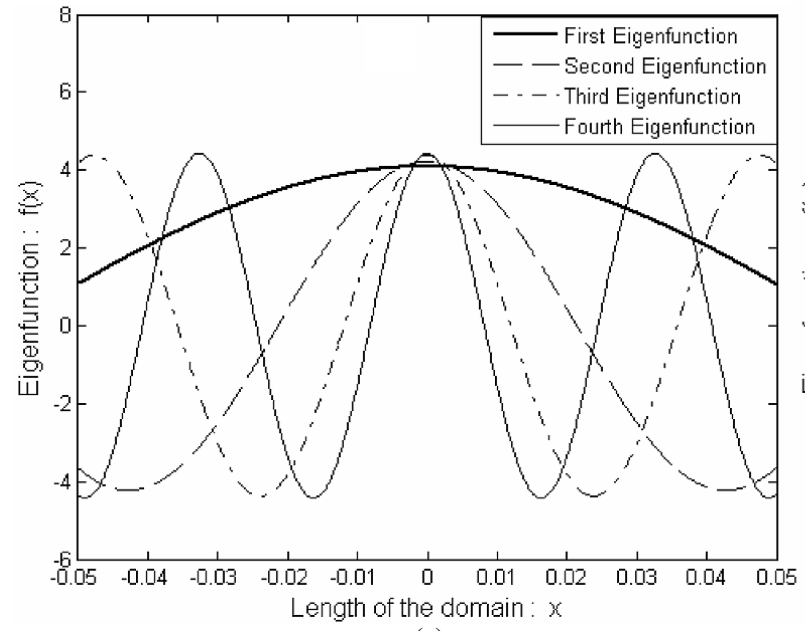

(a)

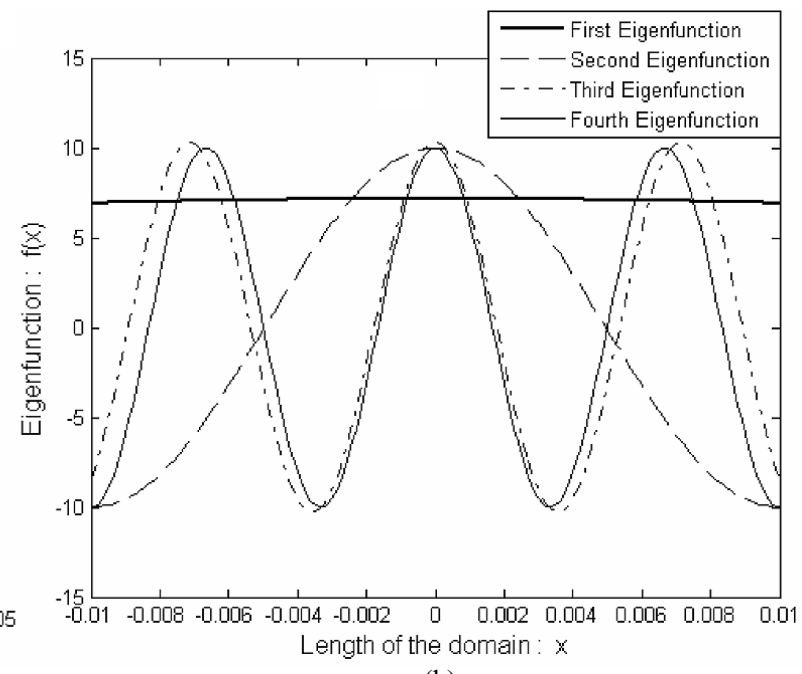

(b)

Fig. 2. Graphs of the first four eigenfunctions of the covariance function: (a) $L_{\text {cor }, x}=0.05 m$ and $\Omega_{x}=[-0.05,0.05] ;(b) L_{c o r, x}=0.01 m$ and $\Omega_{x}=[-0.01,0.01]$.

The expansion detailed previously has been chosen in order to model the elementary random mass and elastic and viscoelastic stiffness matrices of the sandwich plate, as follows:

$$
\begin{aligned}
& \boldsymbol{M}^{(e)}(\theta)=\boldsymbol{M}^{(e)}+\sum_{r=1}^{n} \overline{\boldsymbol{M}}_{r}^{(e)} \xi_{r}(\theta) \\
& \boldsymbol{K}_{e}^{(e)}(\theta)=\boldsymbol{K}_{e}^{(e)}+\sum_{r=1}^{n} \overline{\boldsymbol{K}}_{e_{r}}^{(e)} \xi_{r}(\theta) \\
& \boldsymbol{K}_{v}^{(e)}(\omega, T, \theta)=G(\omega, T) \overline{\boldsymbol{K}}_{v}^{(e)}+G(\omega, T, \theta) \sum_{r=1}^{n} \overline{\boldsymbol{K}}_{v_{r}}^{(e)} \xi_{r}(\theta)
\end{aligned}
$$

where $\boldsymbol{M}^{(e)}, \boldsymbol{K}_{e}^{(e)}$, and $\boldsymbol{K}_{v}^{(e)}(\omega, T)=G(\omega, T) \overline{\boldsymbol{K}}_{v}^{(e)}$, are the mean matrices computed according to Eqs (9), and the random matrices are computed as follows:

$$
\begin{aligned}
& \overline{\boldsymbol{M}}_{r}^{(e)}=\sum_{k=1}^{3} \xi_{r}^{k}(\theta) \int_{x=0}^{x=a} \int_{y=0}^{y=b} \sqrt{\lambda_{r}} f_{r}(x, y) \boldsymbol{N}^{T}(x, y) \boldsymbol{N}(x, y) d y d x \\
& \overline{\boldsymbol{K}}_{e_{r}}^{(e)}=\sum_{k=1,3} \xi_{r}^{k}(\theta) \int_{x=0}^{x=a} \int_{y=0}^{y=b} \sqrt{\lambda_{r}} f_{r}(x, y) \boldsymbol{D}_{k}^{T}(x, y) \boldsymbol{C}_{k} \boldsymbol{D}_{k}(x, y) d y d x
\end{aligned}
$$




$$
\overline{\boldsymbol{K}}_{v_{r}}^{(e)}=\xi_{r}^{v}(\theta) \int_{x=0}^{x=a} \int_{y=0}^{a=b} \sqrt{\lambda_{r}} f_{r}(x, y) \mathbf{D}_{v}^{T}(x, y) \overline{\boldsymbol{C}}_{v} \boldsymbol{D}_{v}(x, y) d y d x
$$

or, equivalently:

$$
\begin{aligned}
& \overline{\boldsymbol{M}}_{r}^{(e)}=\sum_{k=1}^{3} \xi_{r}^{k}(\theta) \alpha_{i} \alpha_{j} \sqrt{\lambda_{i}} \sqrt{\lambda_{j}} \int_{x=0}^{x=a} f_{i}(x) d x \int_{y=0}^{y=b} f_{j}(y) \boldsymbol{N}^{T}(x, y) \boldsymbol{N}(x, y) d y \\
& \overline{\boldsymbol{K}}_{e_{r}}^{(e)}=\sum_{k=1,3} \xi_{r}^{k}(\theta) \alpha_{i} \alpha_{j} \sqrt{\lambda_{i}} \sqrt{\lambda_{j}} \int_{x=0}^{x=a} f_{i}(x) d x \int_{y=0}^{y=b} f_{j}(y) \boldsymbol{D}_{k}^{T}(x, y) \boldsymbol{C}_{k} \boldsymbol{D}_{k}(x, y) d y \\
& \overline{\boldsymbol{K}}_{v_{r}}^{(e)}=\xi_{r}^{v}(\theta) \alpha_{i} \alpha_{j} \sqrt{\lambda_{i}} \sqrt{\lambda_{j}} \int_{x=0}^{x=a} f_{i}(x) d x \int_{y=0}^{y=b} f_{j}(y) \boldsymbol{D}_{v}^{T}(x, y) \overline{\boldsymbol{C}}_{v} \boldsymbol{D}_{v}(x, y) d y
\end{aligned}
$$

In practical applications of surface viscoelastic treatments, the uncertain parameters are primarily those related to the thicknesses of the viscoelastic and constraining layers, and the temperature of the viscoelastic material. The choice of these parameters as the relevant random variables is based on previous knowledge of the fact that they are those that influence the most the performance of the viscoelastic damping treatments, as demonstrated by reference [16]. However, as the temperature does not appear explicitly in the structural matrices, the corresponding uncertainties are introduced in a different way, by using the relation $T(\theta)=T_{0}+T_{0} \delta_{T} \xi(\theta)$, where $T_{0}, \delta_{T}$ and $\xi(\theta)$ designate the temperature mean value, the dispersion level and a Gaussian random variable, respectively.

By combining expressions (15) and (16), and after the standard FE matrix assembling, the frequency domain random equations of motion for the stochastic viscoelastic structure subjected to a deterministic harmonic excitation can be expressed as:

$$
\left[\boldsymbol{K}_{e}(\theta)+G(\omega, T, \theta) \overline{\boldsymbol{K}}_{v}(\theta)-\omega^{2} \boldsymbol{M}(\theta)\right] \boldsymbol{Q}(\omega, T, \theta)=\boldsymbol{F}(\omega)
$$

where $\boldsymbol{M}(\theta), \boldsymbol{K}_{e}(\theta)$ and $\overline{\boldsymbol{K}}_{v}(\theta)$ are the global random mass, and elastic and viscoelastic stiffnesses matrices, respectively, and $\boldsymbol{Q}(\omega, T, \theta)$ designates the stochastic response.

The stochastic viscoelastic problem Eq. (18) must be solved by using a stochastic solver. With this aim, and according to the purposes of this paper, MCS in combination with LHC sampling method is used. In addition, the probability distributions of the uncertainty variables are chosen a priori.

\subsection{Condensation of the stochastic viscoelastic model}

In most cases of industrial interest, it becomes practically impossible to compute the random FRFs directly from Eq. (18), owing to the prohibitive amount of computation and storage memory required, in addition to the large number of computations of the HCL samples required to evaluate the FRFs variability with assured convergence. Those difficulties motivate the use of model condensation procedures, which aim at reducing the model dimensions and the associated computational cost, while keeping a reasonable predictive capacity of the numerical stochastic viscoelastic models. This can be done based on the assumption that the exact responses, given by the resolution of Eq. (18) for each sample $\theta$, can be approached by the projection of the response vector on a reduced vector basis as follows:

$$
\boldsymbol{Q}(\omega, T, \theta)=\boldsymbol{T} \hat{\boldsymbol{Q}}(\omega, T, \theta)
$$

where $\boldsymbol{T} \in C^{N \times N R}$ is the transformation matrix formed column-wise by a vector basis, $\hat{\boldsymbol{Q}}(\omega, T, \theta) \in C^{N R}$ are generalized coordinates, and $N R<<N$ is the number of reduced vectors in the basis. 


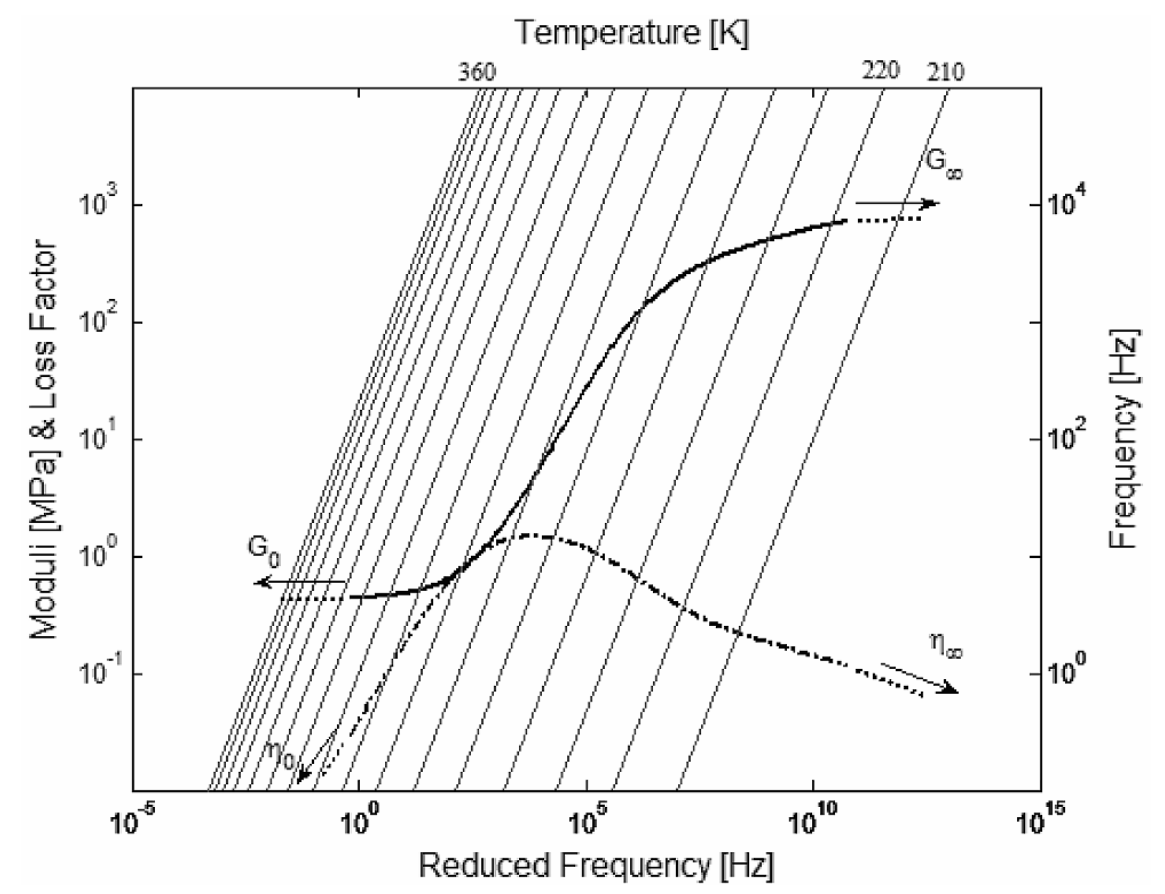

Fig. 3. Illustration of the asymptotic properties of storage modulus and loss modulus.

By considering Eqs (18) and (19), the stochastic receptance matrix can be rewritten as:

$$
\hat{\boldsymbol{H}}(\omega, T, \theta)=\left[\boldsymbol{T}^{T} \boldsymbol{K}_{e}(\theta) \boldsymbol{T}+G(\omega, T, \theta) \boldsymbol{T}^{T} \overline{\boldsymbol{K}}_{v}(\theta) \boldsymbol{T}-\omega^{2} \boldsymbol{T}^{T} \boldsymbol{M}(\theta) \boldsymbol{T}\right]^{-1}
$$

For models containing viscous or structural damping, it is relatively common to use a constant projection basis formed by the eigenvectors of the associated conservative structure, as the mass and stiffness matrices are invariant [10]. However, for viscoelastic systems, the selection of the reduction basis is more delicate as this condition does not hold. Owing to the dependence of the stiffness matrix with respect to frequency, the reduction basis should be able to represent the changes of the dynamic behavior as frequency is varied in the band of interest [23]. In this work, the strategy proposed consists in using a reduction basis formed by a constant modal basis of the associate conservative system. However, this basis is enriched by static residual vectors to account for the effects of the external loads and the viscoelastic damping forces. These static responses are computed from the low-frequency asymptotic stiffness matrix, representing the static behavior of the viscoelastic materials, which is given by:

$$
\boldsymbol{K}_{0}=\boldsymbol{K}_{e}+G_{0} \overline{\boldsymbol{K}}_{v}
$$

where $G_{0}$ is the real part of the modulus function obtained by the extrapolation of the modulus curves in the low frequency range by asymptotes, as shown in Fig. 3.

Then, the nominal basis can be obtained by the resolution of the eigenvalue problem:

$$
\begin{aligned}
& \left(\boldsymbol{K}_{0}-\lambda_{i} \boldsymbol{M}\right) \varphi_{i}=0 \quad i=1, \ldots, N \\
& \boldsymbol{\varphi}_{0}=\left[\begin{array}{ll}
\varphi_{1} \varphi_{2} \ldots \varphi_{N R}
\end{array}\right], \quad \Lambda_{0}=\operatorname{diag}\left(\lambda_{1}, \ldots, \lambda_{N R}\right)
\end{aligned}
$$

In general, a relatively large number of eigenvectors must be kept in the projection basis to guarantee the accuracy of the reduced model. It has been shown in previous studies [23] that the number of necessary projection vectors can be reduced by introducing the residues formed by the static displacements associated to external forces, $R=K_{0}^{-1} \boldsymbol{b}$, where $\boldsymbol{b} \in R^{N \times f}$ is Boolean matrix which enable to select, among the DOFs, those in which the excitation forces are applied, and the residues associated to the viscoelastic damping forces, $\boldsymbol{R}_{v}^{0}=\boldsymbol{K}_{0}^{-1} \overline{\boldsymbol{K}}_{v} \boldsymbol{\varphi}_{0}$. As detailed in references $[23,24]$, these residuals are interpreted as the columns of the flexibility matrix of the undamped system, 
associated to the coordinates of application of two types of forces: the external excitation forces and the damping forces. These latter can be better understood by examining Eq. (18), noting that the term involving the viscoelastic behavior can be moved to the right-hand side, where it plays the role of additional forces applied to the associated conservative structure. Thus, the enriched basis of reduction for the viscoelastic system is given as follows:

$$
\boldsymbol{T}=\left[\begin{array}{lll}
\boldsymbol{\varphi}_{0} & \boldsymbol{R} & \boldsymbol{R}_{v}^{0}
\end{array}\right]
$$

\section{Numerical applications}

\subsection{First test-structure: rectangular plate partially treated by PCLD}

The first test-structure is a simple rectangular plate, whose geometrical features and FE mesh are depicted in Fig. 4. The FE element model without viscoelastic treatment is composed by 143 elements comprising a total number of 1185 DOFs. The surface viscoelastic treatment (named herein Passive Constrained Layer Damping - PCLD), whose position and extent have been chosen arbitrarily, is indicated by the shadowed areas, comprising two zones and a total number of 36 finite elements. Free boundary conditions are assumed. Table 1 presents the values of the geometrical and mechanical characteristics of the plate, as well as the uncertain parameters with the corresponding assumed dispersion levels. The values of the material properties for the base-plate and constraining layer are: Young modulus, $E_{1}=E_{3}=70 \mathrm{GPa}$; Poisson ratio, $\nu_{1}=\nu_{3}=0.34$; and mass density, $\rho_{1}=\rho_{3}=2700 \mathrm{~kg} / \mathrm{m}^{3}$. For the viscoelastic core, the modulus function of the commercially available 3M ISD112 ${ }^{\circledR}$ viscoelastic material $\left(\rho_{2}=\right.$ $950 \mathrm{~kg} / \mathrm{m}^{3}, \nu_{2}=0.49$ ) represented by Eqs (3), Section 2, is adopted.

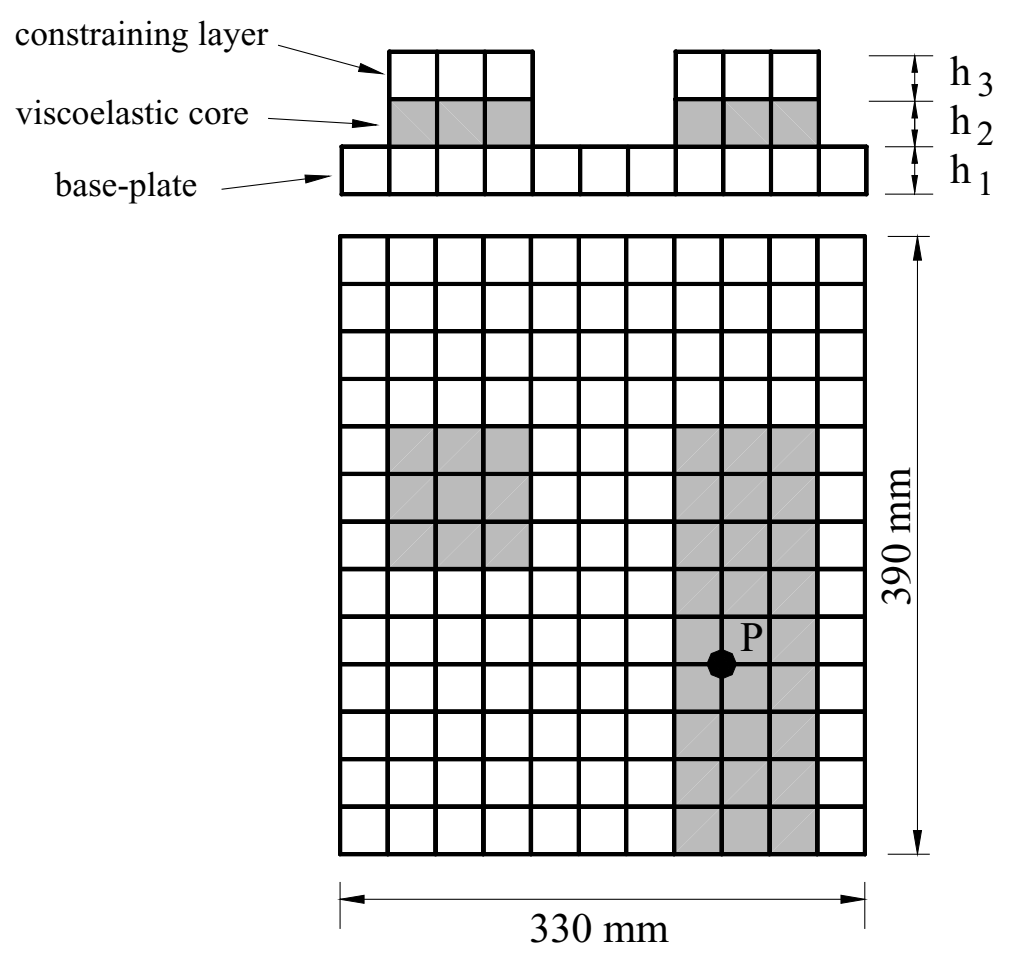

Fig. 4. FE model of the plate treated with constrained damping layers.

In a first step, one is interested in verifying the accuracy of the reduced model of the viscoelastic system based on Eqs (20) and (23). The computations consist in obtaining the driving point FRF $\hat{H}(\omega, T)$ in the frequency band [20-110 Hz], comprising the first four vibration modes, corresponding to the transverse displacement at point $P$, 
Table 1

Uncertainty levels for the plate

\begin{tabular}{lccccc}
\hline Random variables & Nominal values & \multicolumn{4}{c}{ Uncertainty level } \\
\cline { 3 - 6 } & & Case 1 & Case 2 & Case 3 & Case 4 \\
\hline $\mathrm{T}$ & $25^{\circ} \mathrm{C}$ & --- & --- & -- & $10 \%$ \\
$h_{1}$ & $1.5 \mathrm{~mm}$ & --- & $5 \%$ & $10 \%$ & $10 \%$ \\
$h_{2}$ & $0.0254 \mathrm{~mm}$ & $5 \%$ & $5 \%$ & $10 \%$ & $10 \%$ \\
$h_{3}$ & $0.50 \mathrm{~mm}$ & $5 \%$ & $5 \%$ & $10 \%$ & $10 \%$ \\
\hline
\end{tabular}

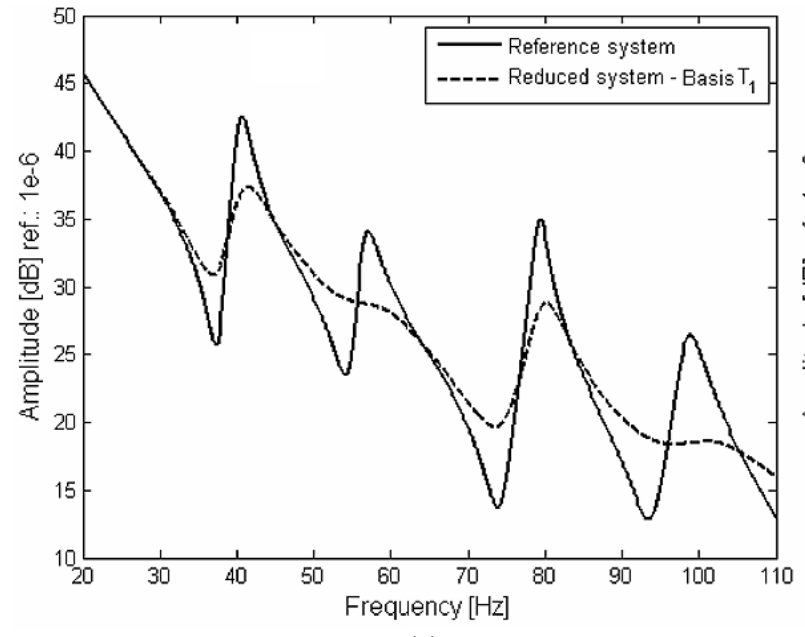

(a)

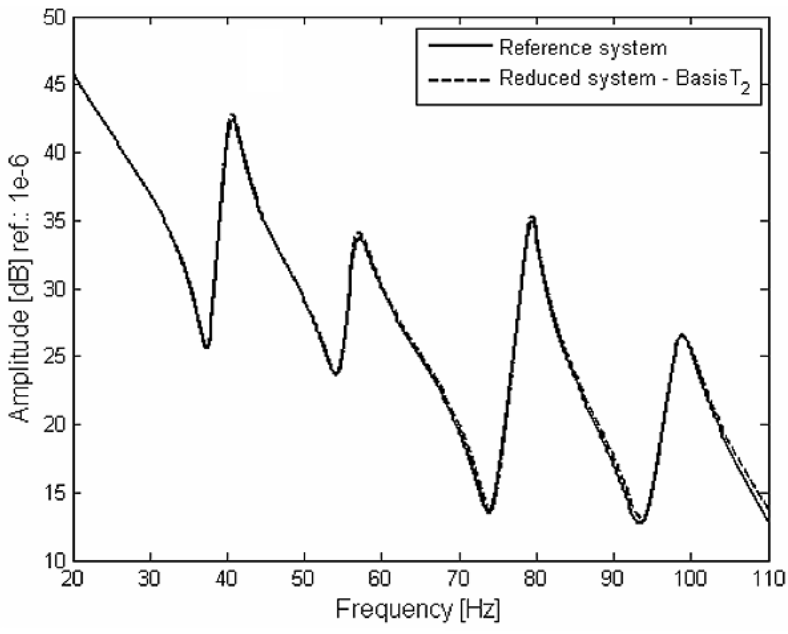

(b)

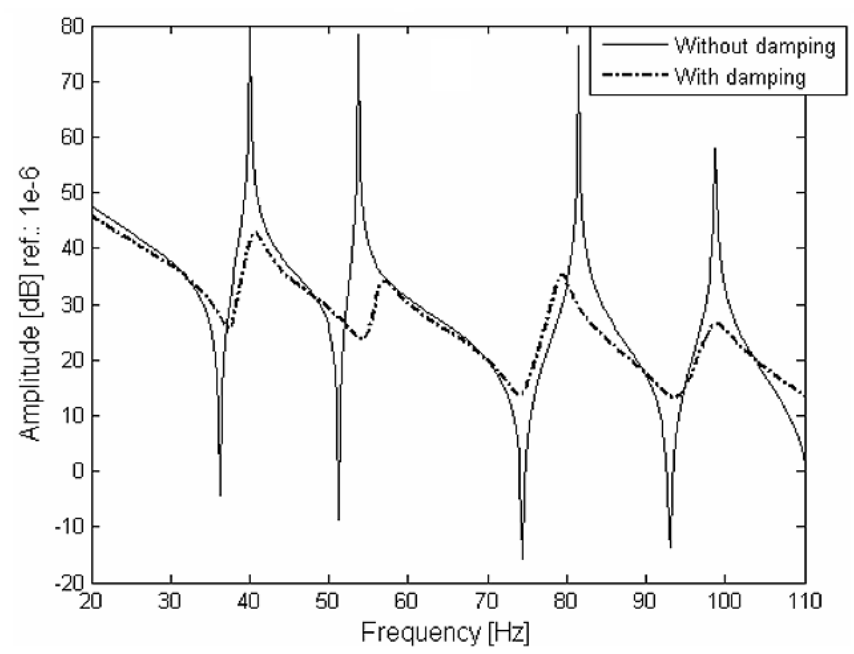

(c)

Fig. 5. FRF amplitudes of the reference and reduced systems by using reduction bases. (a) $T_{1}$ and (b) $T_{2}$. (c) FRF amplitudes obtained for the plate with and without PCLD.

the position of which has been chosen arbitrary, being indicated in Fig. 4. It is assumed that the temperature of the viscoelastic material is $25^{\circ} \mathrm{C}$, constant and uniform. One considers two nominal bases: (a) $\boldsymbol{T}_{1}=\left[\begin{array}{ll}\phi_{0} & R\end{array}\right](30$ eigenvectors, plus one residual vector associated to the external force, computed by the relation $\boldsymbol{R}=\boldsymbol{K}_{0}^{-1} \boldsymbol{b}$ ); (b) $\boldsymbol{T}_{2}=\left[\begin{array}{llll}\phi_{0} & \boldsymbol{R} & \boldsymbol{R}_{v}^{0}\end{array}\right]$ (30 eigenvectors, one residual vector computed by the relation $\boldsymbol{R}=\boldsymbol{K}_{0}^{-1} \boldsymbol{b}$, and 19 residual vectors associated to the damping forces, computed according to the relation $\boldsymbol{R}_{v}^{0}=\boldsymbol{K}_{0}^{-1} \overline{\boldsymbol{K}}_{v} \boldsymbol{\phi}_{0}$ ). These bases have been computed from the model corresponding to the nominal parameter values presented in Table 1.

Figures 5(a) and 5(b) show the amplitudes of the FRF's computed by using the two bases, as compared to the 
amplitudes of the FRF computed by using a reference basis formed by a far larger number of eigenvectors (100) and residual vectors (100). It can be clearly seen that the use of first order residues associated with the viscoelastic damping forces are indispensable for the accurate representation of the dynamic behavior of the viscoelastic damped system into the frequency band of interest. Additionally, Fig. 5(c) presents the FRFs of the plate with and without viscoelastic treatments (computed by using the large reference basis) and enables to evaluate the effectiveness of the viscoelastic patches for mitigating the response levels.

To provide a sense of the reduction of the computation effort obtained with the use of the proposed model condensation strategy, Table 2 gives the comparison between the time required to compute a single FRF in the frequency band of interest, for both the reduced model Eq. (20), using the basis $\boldsymbol{T}_{2}$ previously defined, and for the full FE model Eq. (18). It becomes obvious the reduction of computation time achieved by the use of the reduced model. In addition, it must be remembered that in the evaluation of a large number of samples one can take advantage of the parameterization scheme presented in Section 4 for expediting the actualization of the structural matrices and reassembling prior to reanalysis.

Table 2

Time to compute the FRF's for the reduced and complete plate models

\begin{tabular}{lcc}
\hline & Reduced model & Full model \\
\hline Matrix assembling & $51 \mathrm{~s}$ & $51 \mathrm{~s}$ \\
Computation of the FRF from Eqs (18) and (20) & $14 \mathrm{~s}$ & $110 \mathrm{~s}$ \\
Total computation time & $65 \mathrm{~s}$ & $161 \mathrm{~s}$ \\
\hline
\end{tabular}

In the sequence, uncertainties are introduced in the random variables with the levels defined in Table 1 . The computations of the stochastic matrices of the sandwich plate are performed by assuming the correlation lengths $L_{c o r, x}=L_{c o r, y}=30 \mathrm{~mm}$, corresponding to the dimension of the sides of the square elements, according to the FE mesh depicted in Fig. 4. For the numerical simulations, convergence analyses were first performed according to reference [13] for the different dispersion levels. It has been verified that the solutions always converge for 500 LHC samples. Figure 6 illustrates the envelopes of the random responses of the viscoelastic system for the dispersion levels reported in Table 1. In particular, from Figs 6(a), 6(b), and 6(c), it is possible to observe that as the parameter dispersion levels increase, the response dispersions increase accordingly, being larger for higher frequencies. In addition, by comparing Figs 6(a) and 6(b) one can observe the degree of influence of the variability of the thickness of the base-structure. By comparing the results shown in Fig. 6(d), corresponding to Case 4, in which temperature dispersions are considered, with those corresponding to the other cases, one can observe the strong influence of the temperature of the viscoelastic material on the dynamic responses of the viscoelastic structure. The general trend observed is that the dispersion of the responses increase as the number of random variables is increased, as expected. For all the cases, the limits of the samples enclose the dynamic response of the mean model.

\subsection{Second test-structure: stiffened panel treated with PCLD}

Figure 7(a) depicts the second test-structure, which is more complex than the plate considered previously, being composed by a freely suspended stiffened panel containing four stringers. The FE model without viscoelastic treatment is composed by 928 elements having a total number of 5940 DOFs. The viscoelastic treatment, also indicated in Fig. 7(a), is composed by 10 viscoelastic patches, each one comprising 16 three-layer sandwich plate elements. The FE model of the treated panel contains 6840 DOFs. The geometric dimensions are: internal radius: $938 \mathrm{~mm}$; length: $720 \mathrm{~mm}$; radius: $680 \mathrm{~mm}$; thicknesses of the panel and the stringers: $1.5 \mathrm{~mm}$ and $0.75 \mathrm{~mm}$, respectively; height of the stringers: $30 \mathrm{~mm}$. The material properties for both panel and stringers are: Young modulus, $E_{1}=210 \mathrm{GPa}$; mass density, $\rho_{1}=780 \mathrm{Kg} / \mathrm{m}^{3}$; Poisson ratio, $\nu_{1}=0.30$. The material properties of the constraining layer and viscoelastic material are the same as those used in the first application. The nominal values of the design parameters and the corresponding uncertainty levels are defined in Table 3. The computation of the envelopes of dynamic responses consisted in obtaining the random driving point FRF $H(\omega, T)$ associated to point $P$ indicated on Fig. 7(a), in the frequency band of interest [146-200 Hz].

To verify the condensation procedure, one considers the following nominal bases: $T_{1}=\left[\phi_{0}\right]$ (60 eigenvectors); $T_{2}=\left[\begin{array}{ll}\phi_{0} & \boldsymbol{R}\end{array}\right]$ (60 eigenvectors, plus one residual vector associated to the external force); $T_{3}=\left[\begin{array}{lll}\phi_{0} & \boldsymbol{R} & \boldsymbol{R}_{v}^{0}\end{array}\right](60$ 
Table 3

Uncertainty levels for the stiffened panel

\begin{tabular}{lccc}
\hline Random variables & Nominal values & \multicolumn{2}{c}{ Uncertainty level } \\
\cline { 3 - 4 } & & Case 1 & Case 2 \\
\hline$T$ & $25^{\circ} \mathrm{C}$ & -- & $5 \%$ \\
$h_{2}$ & $0.0254 \mathrm{~mm}$ & $5 \%$ & $5 \%$ \\
$h_{3}$ & $0.5 \mathrm{~mm}$ & $5 \%$ & $5 \%$ \\
\hline
\end{tabular}

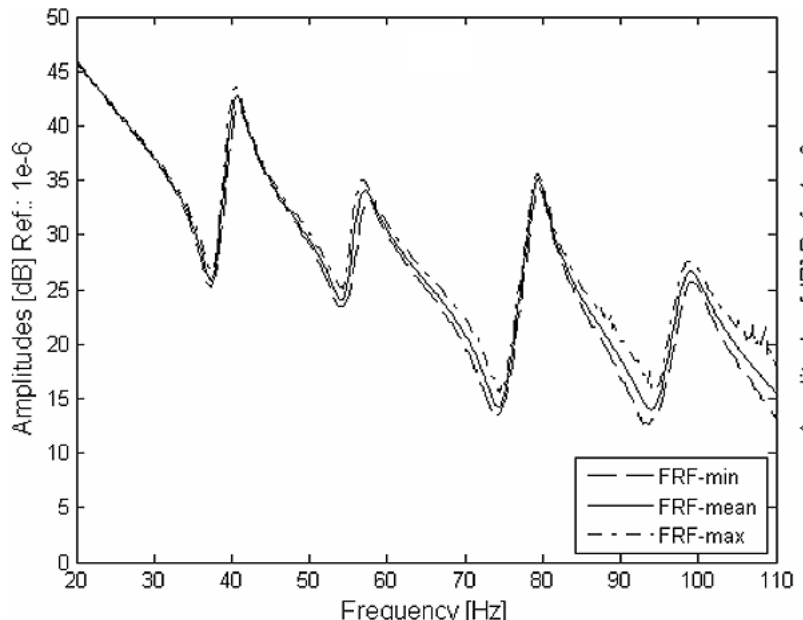

(a)

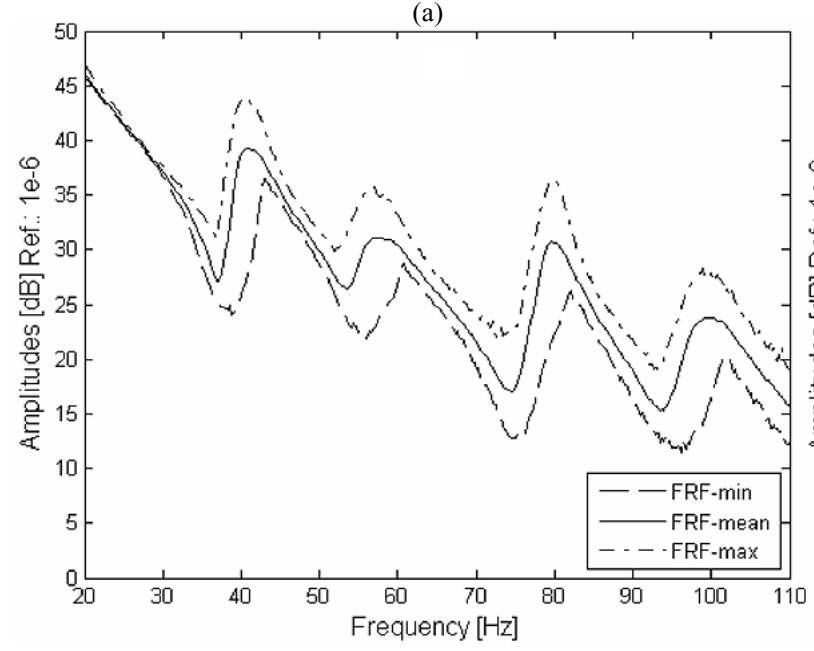

(c)

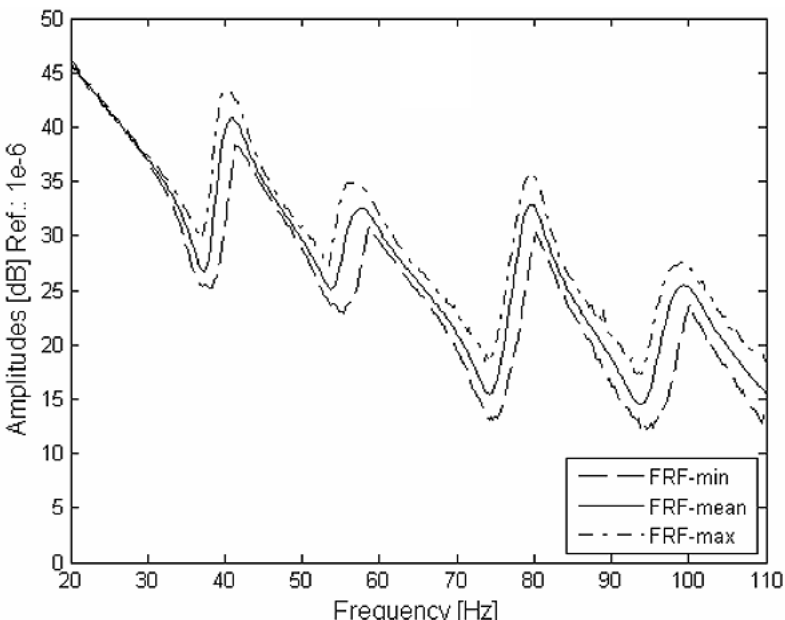

(b)

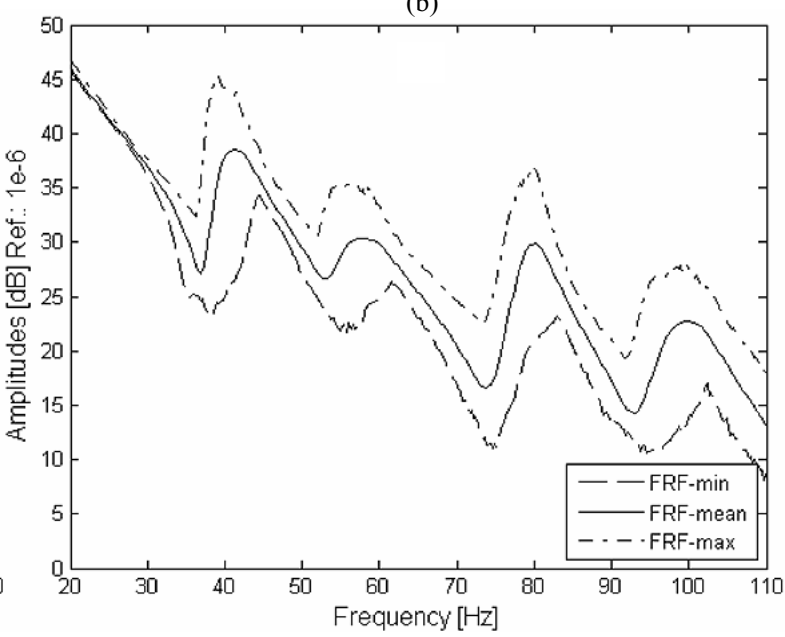

(d)

Fig. 6. Envelopes of random FRFs of the plate treated with constrained layers for uncertainty levels: (a) Case 1; (b) Case 2; (c) Case 3; (d) Case 4.

eigenvectors, one residual vector associated to the external force, 54 residual vectors associated to the damping forces). Figures 7(b), 7(c) and 7(d) show the amplitudes of the FRFs computed by using the three bases, as compared to the amplitudes of the FRF computed by using a reference basis formed by a far larger basis composed of 600 eigenvectors and 600 residual vectors. It can be clearly seen that the accuracy is continuously improved upon successive enrichment of the reduction basis by the inclusion of residual vectors accounting for the static residues associated to the external loads and damping forces, to form the basis $T_{2}$ and $T_{3}$, respectively, and that, again, the inclusion of the residual vector associated to the damping forces provide the necessary accuracy of the reduced model.

Figures 8(a) and 8(b) illustrate the random FRFs obtained the two cases reported in Table 3, in which the random 
Table 4

Time to compute the FRF's for the reduced and complete stiffened panel models

\begin{tabular}{lcc}
\hline & Reduced model & Full model \\
\hline Matrix assembling & $86 \mathrm{~s}$ & $86 \mathrm{~s}$ \\
Computation of the FRF from Eqs (18) and (20) & $17 \mathrm{~s}$ & $730 \mathrm{~s}$ \\
Total computation time & $103 \mathrm{~s}$ & $816 \mathrm{~s}$ \\
\hline
\end{tabular}

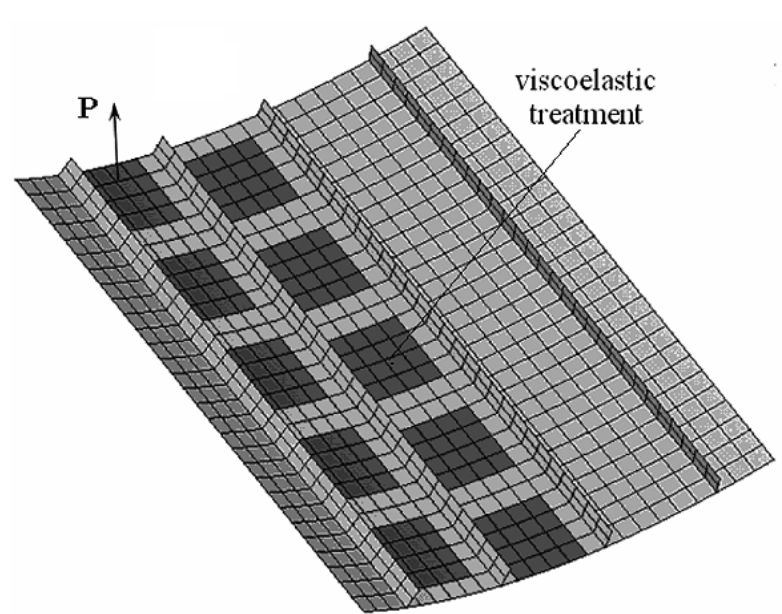

(a)

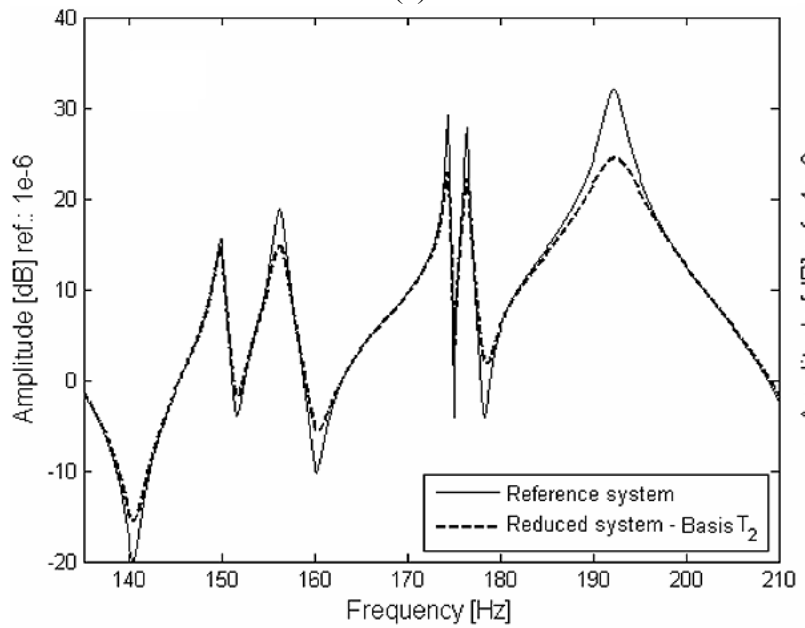

(c)

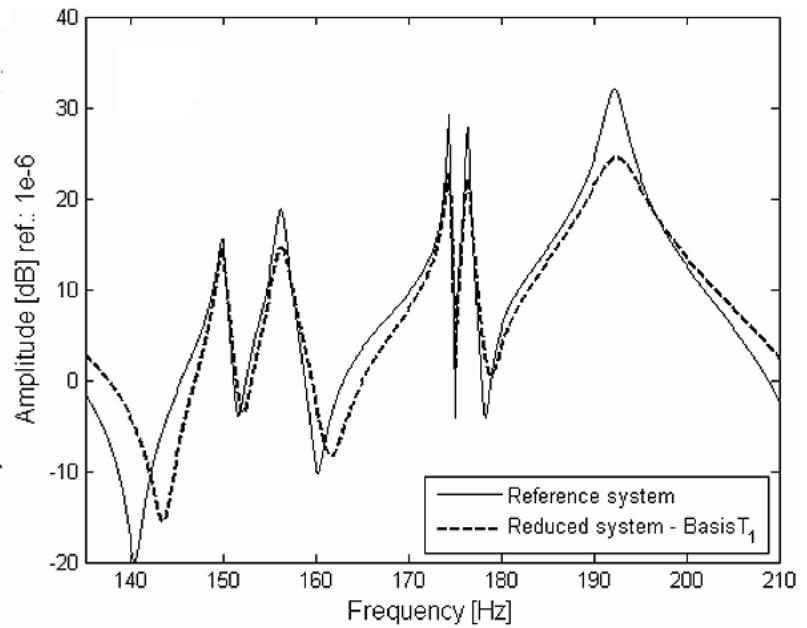

(b)

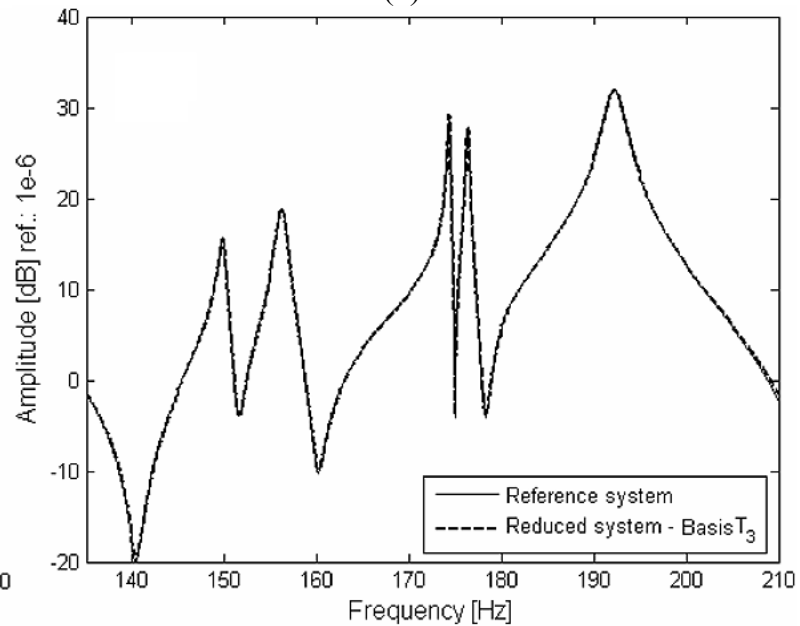

(d)

Fig. 7. (a) FE model of the stiffened panel treated with PCLD; FRF amplitudes of the reference and reduced systems by using the basis (b) $T_{1}$, (c) $T_{2}$ and (d) $T_{3}$.

variables and corresponding dispersions are presented. Similarly to what has been observed in the previous example, when the temperature is taken as uncertain, the dispersion of the responses becomes larger. Once again, the trend of increased dispersions for higher frequencies is noticed.

Table 4 provides the comparison between the time required to compute a single FRF in the frequency band of interest, for the reduced model, using the basis $\boldsymbol{T}_{3}$ and the full FE model, which leads to conclude that for largescale viscoelastic systems, the computation of the FRFs variability based on the full stochastic matrices is virtually unfeasible and that the used of the suggested model reduction procedure can make such computations affordable. 


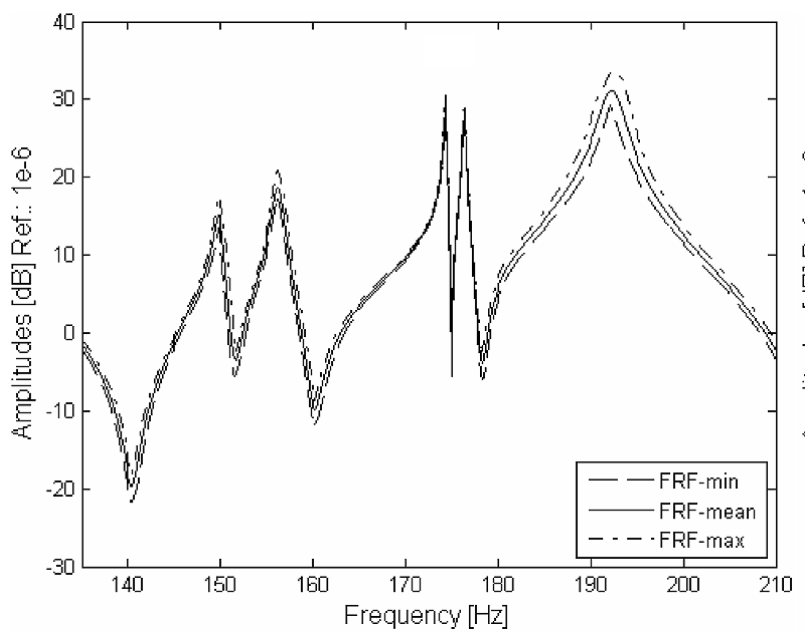

(a)

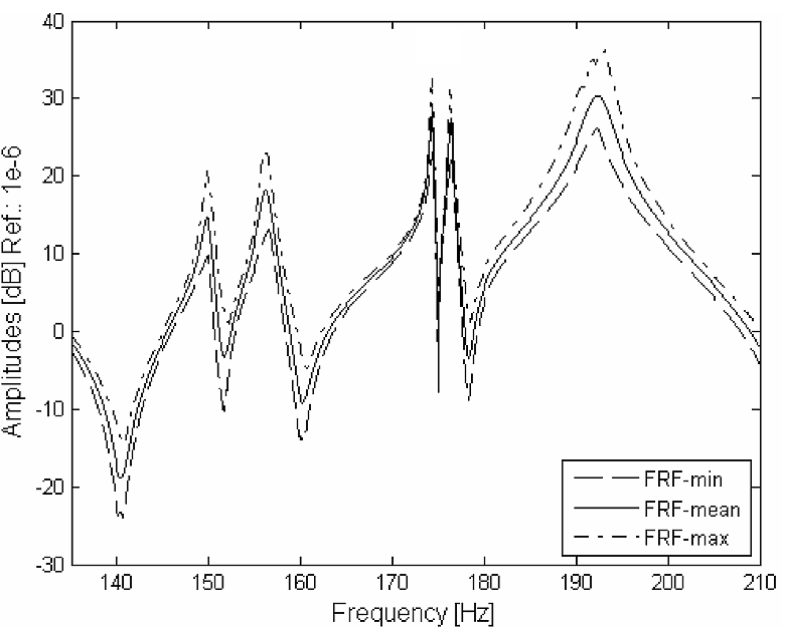

(b)

Fig. 8. Envelopes of random FRFs of the damped stiffness panel for uncertainty levels: (a) Case 1, (b) Case 2.

\section{Concluding remarks}

A modeling strategy for uncertainty propagation based on the stochastic finite element approach combined with a model reduction technique specially adapted to structural systems containing viscoelastic surface treatments has been suggested, accounting for the dependence of the stiffness matrix with respect to frequency and temperature through the concept of complex modulus. The uncertainties assigned to the design parameters and also to the temperature are introduced after an adequate parameterization of the mass and complex stiffness matrices. Numerical simulations demonstrate that the suggested strategy enables to perform uncertainty propagation through complex structural models, achieving significant computational cost savings and, at the same time, preserving the predictive capacity of the models.

\section{Acknowledgments}

The authors are grateful to the following organizations: (i) Brazilian Research Council - CNPq for the continued support to their research work, especially through research project 480785/2008-2 (A.M.G. de Lima) and research grant 310524/2006-7 (D.A. Rade); (ii) Minas Gerais State Agency FAPEMIG for the financial support to their research activities; (iii) CAPES Foundation, from the Brazilian Ministry of Education.

\section{References}

[1] D.F. Golla and P.C. Hughes, Dynamics of viscoelastic structures - A time domain, finite element formulation, Journal of Applied Mechanics 52(4) (1985), 897-906.

[2] G.A. Lesieutre and E. Bianchini, Time domain modeling of linear viscoelasticity using anelastic displacement fields, ASME Journal 117(4) (1995), 424-430.

[3] R.L. Bagley and P.J. Torvik, A generalized derivative model for an elastomer damper, Shock and Vibration Bulletin 49(2) (1979), $135-143$.

[4] D.J. McTavish, P.C. Hughes, Y. Soucy and W.B. Graham, Prediction and measurement of modal damping factors for viscoelastic space structures, AIAA Journal 30(5) (1992), 1392-1399.

[5] D. Ottl, Beschreibung linear-viskoelastischer schwingungssysteme mit hilfe von inner variablen, ZAMM-Zeitschrift fuer Angewandte Mathematik und Mechanik 67(4) (1987), T122-T124 (in German).

[6] I. Elishakoff, P. Elisseeff and S.A.L. Glegg, Nonprobabilistic, convex-theoretic modeling of scatter in material properties, AIAA Journal 32(4) (1994), 843-849.

[7] F.A. Cozzarelli and W.N. Huang, Effect of random material parameters on nonlinear steady creep solutions, International Journal of Solids and Structures 7 (1971), 1477-1494. 
[8] W.N. Huang and F.A. Cozzarelli, Steady creep in a beam with random material parameters, Journal of the Franklin Institute 294(5) (1972), 323-338.

[9] H.H. Hilton, J. Hsu and J.S. Kirby, Linear viscoelastic analysis with random material properties, Stochastic Structural Dynamics 1 - New Theoretical Developments, Springer-Verlag, Berlin, 83-110, 1991.

[10] A.D. Nashif, D.I.G. Jones and J.P. Henderson, Vibration Damping, John Wiley \& Sons, New York, 1985.

[11] R.G. Ghanem and P.D. Spanos, Stochastic finite elements: A spectral approach, Spring Verlag, 1991.

[12] B.V. Nieuwenhof and J.P. Coyette, Modal approaches for the stochastic finite element analysis of structures with material and geometric uncertainties, Computer Methods in Applied Mechanics and Engineering 192(33) (2003), 3705-3729.

[13] R. Sampaio and C. Soize, 2007, On measures of nonlinearity effects for uncertain dynamical systems applications to a vibro-impact system, Journal of Sound and Vibration 303(3) (2007), 659-674.

[14] M. Guedri, A.M.G. de Lima, N. Bouhaddi and D.A. Rade, Robust design of viscoelastic structures based on stochastic finite element models, Mechanical System and Signal Processing 24(1) (2010), 59-77.

[15] A. Florian, 1992, An efficient sampling scheme: updates Latin Hypercube sampling, Probabilistic Engineering Mechanics 7(2) (1992), $123-130$.

[16] A.M.G de Lima, M.H. Stoppa, D.A. Rade and V. Jr. Steffen, Sensitivity Analysis of Viscoelastic Systems, Shock and Vibration 13(4) (2006), 545-558

[17] A.M.G. de Lima, D.A. Rade and F.P. Lépore-Neto, An efficient modeling methodology of structural systems containing viscoelastic dampers based on frequency response function substructuring, Mechanical System and Signal Processing 23(4) (2009), 1272-1281.

[18] A.M.G. de Lima, A.W. Faria and D.A. Rade, Sensitivity analysis of frequency response functions of composite sandwich plates containing viscoelastic layers, Composite Structures 92(1) (2010), 364-376.

[19] A. Moreau, Identification de propriétés viscoélastiques de matériaux polymères par mesures de réponses en fréquences, Ph.D. Thesis, INSA-Rouen, 2007 (in French).

[20] M.L. Drake and J. Soovere, A Design Guide for Damping of Aerospace Structures, AFWAL Vibration Damping Workshop Proceedings, Atlantic City, USA, 1984.

[21] http://www.3m.com/ (Accessed 01 August 2008).

[22] T.P. Khatua and Y.K. Cheung, Bending and Vibration of Multilayer Sandwich Beams and Plates, International Journal for Numerical Methods in Engineering 6(1) (1973), 11-24.

[23] A.M.G. Lima, 2007, "Modeling and Robust Optimization of Viscoelastic Damping in Mechanical Systems", Doctorate Thesis, University of Franche-Comté (UFC), Besançon in France, and Federal University of Uberândia (UFU), Uberlândia in Brazil (in French).

[24] E. Balmès and S. Germès, Tools for viscoelastic damping treatment design: Application to an automotive floor panel, Proceedings of the 28th International Seminar on Modal Analysis, Leuven, September, 2002. 

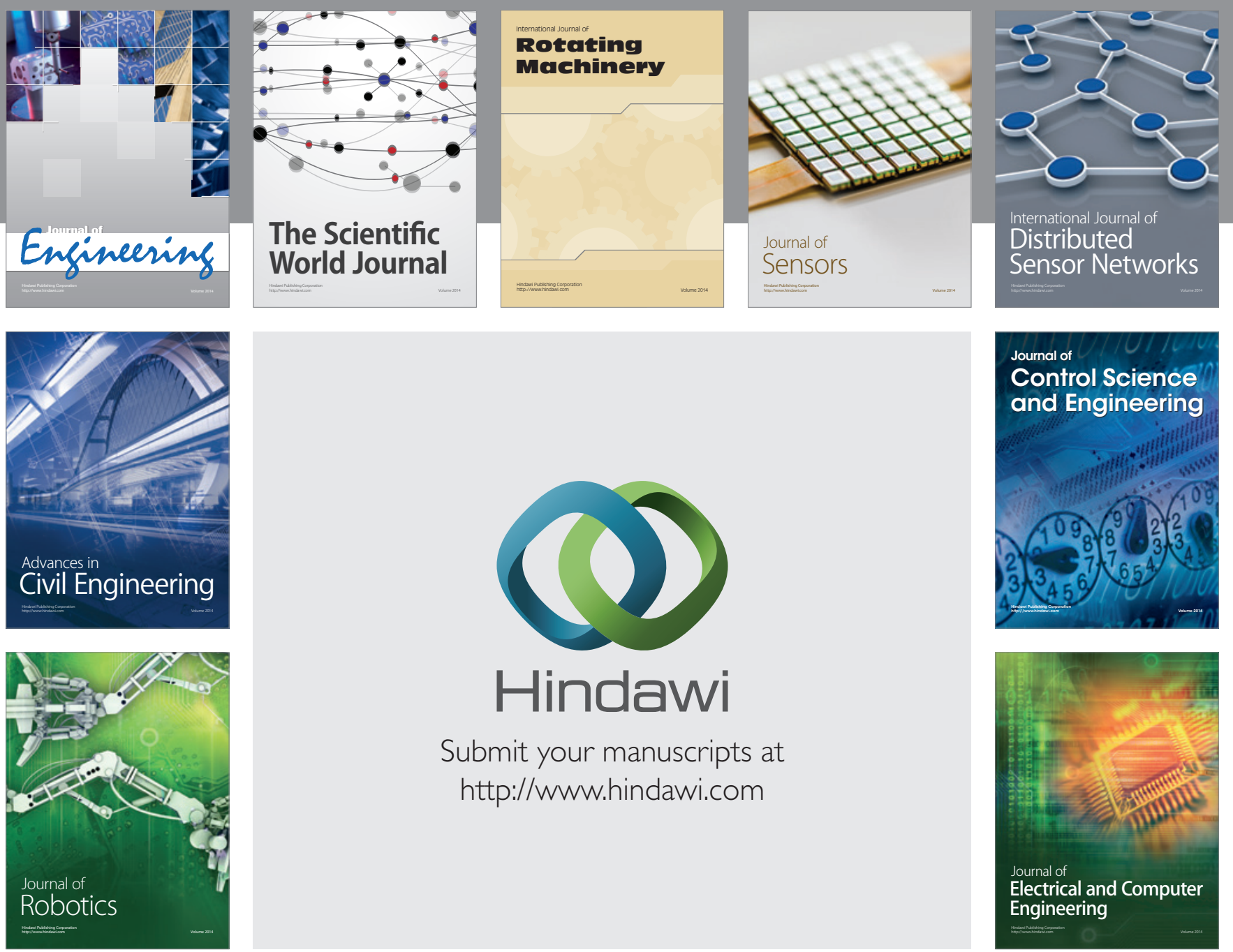

Submit your manuscripts at

http://www.hindawi.com
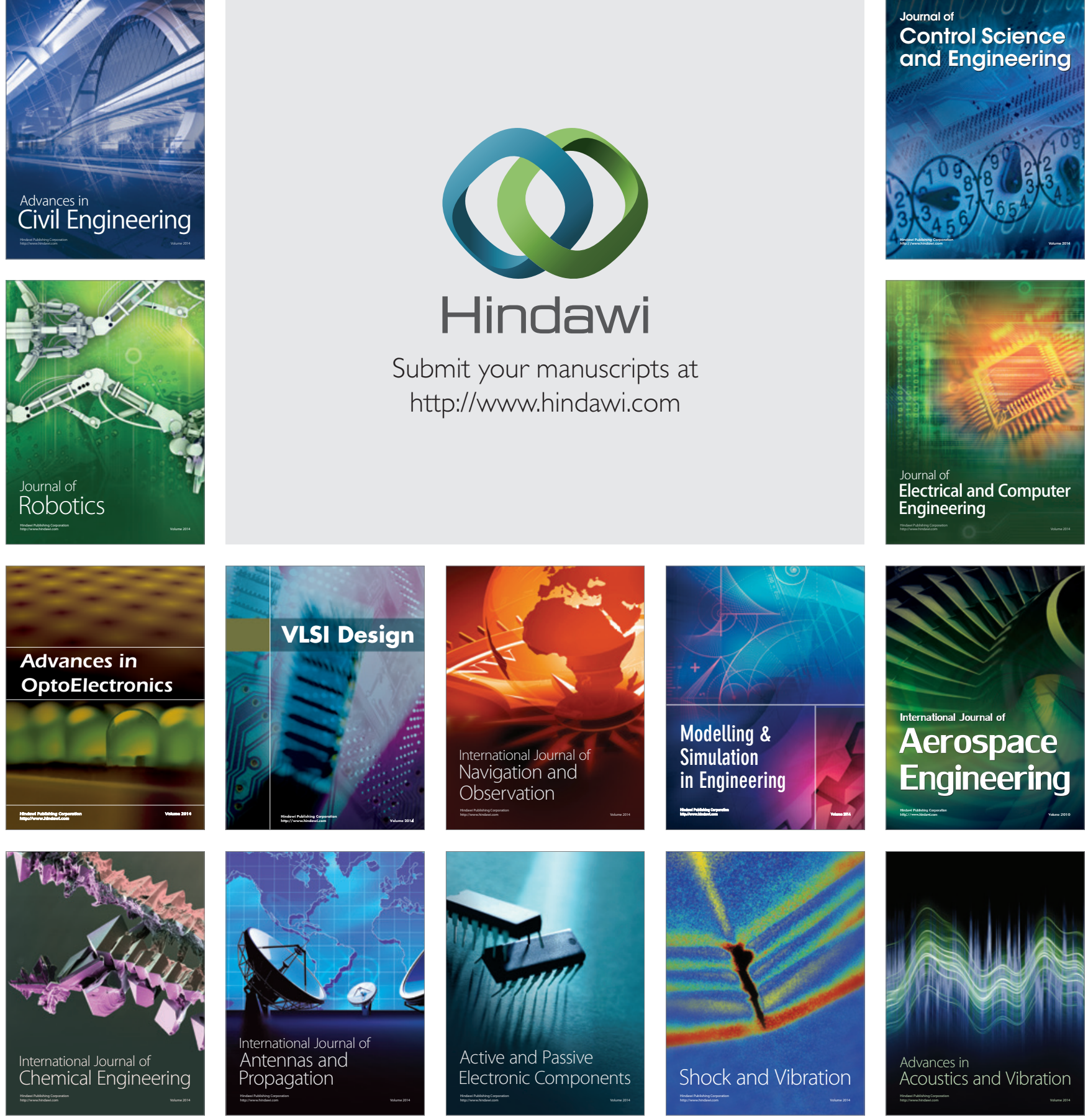